\title{
Réplica: Sobre la evaluación del clima organizacional y satisfacción del usuario en el Instituto Nacional de Salud del Niño
}

\section{Reply: On the evaluation of the organizational climate and user satisfaction at the Instituto Nacional de Salud del Niño}

\author{
Danitza Fernández-Oliva ${ }^{1,2, a}$, María E. Revilla-Velásquez ${ }^{1,2, a}$, Víctor A. Mamani-Urrutia ${ }^{1,3, b}$ \\ Instituto Nacional de Salud del Niño. Lima, Perú. \\ 2 Facultad de Medicina, Universidad Nacional Mayor de San Marcos. Lima, Perú. \\ ${ }^{3}$ Facultad de Ciencias de la Salud, Universidad Científica del Sur. Lima, Perú. \\ a Médico pediatra

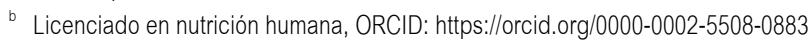

An Fac med. 2020;81(1):129-30. / https://doi.org/10.15381/anales.v81i1.17789

\begin{abstract}
Correspondencia:
Víctor Alfonso Mamani Urrutia

vmamaniu@gmail.com

Recibido: 13 de marzo 2020

Aprobado: 23 de marzo 2020

Publicación en línea:31 de marzo 2020

Conflictos de interés: Los autores

declaran no tener conflictos de interés

Fuentes de financiamiento:

Autofinanciado

Citar como: Fernádez-Oliva D, RevillaVelásquez M, Bustamante-López A, Mamani-Urrutia V. Réplica: Sobre la evaluación del clima organizacional y satisfacción del usuario en el Instituto Nacional de Salud. An Fac med. 2020;81(1):129-30. DOI: https://doi. org/10.15381/anales.v81i1.17789
\end{abstract}

\section{Sr. Editor,}

Agradecemos los comentarios al artículo publicado en la revista que dirige. Permítanos primero aclarar algunas dudas y brindar información adicional para facilitar la revisión del artículo. Es necesario indicar que nuestro estudio no tuvo como finalidad correlacionar las variables de clima organizacional -reportado por los trabajadores del Instituto Nacional de Salud del Niño (INSN)- y la satisfacción del usuario externo -reportada por quienes acuden a esta institución especializada de salud-. Para correlacionar estas variables serían necesarios aspectos metodológicos y estadísticos diferentes a los que nos planteamos, como por ejemplo homogenizar la población, considerar variables confusoras y correlacionar pares de datos, entre otros ${ }^{(1,2)}$. Consideramos que este tipo de análisis no es recomendable, aunque se ha utilizado en estudios previos ${ }^{(3-6)}$.

Sobre la población evaluada para determinar la satisfacción del usuario externo, debemos precisar que siendo el INSN un centro pediátrico, se optó en el diseño del estudio por entrevistar a los familiares o acompañantes de los pacientes hospitalizados, quienes viven de cerca los procesos que conlleva la estancia de los pacientes. De ese modo, pueden responder a las preguntas del instrumento planteado para medir satisfacción del usuario externo. Sólo en dos casos se entrevistó a pacientes que tenían la mayoría de edad, como se mostró en la tabla 3 del estudio ${ }^{(7)}$.

Finalmente, aunque ambas variables pueden ser estudiadas por separado, consideramos relevante describirlas juntas, con la finalidad de enfatizar la situación que atraviesa un centro de referencia nacional que atiende por más de 90 años a la población pediátrica más vulnerable del país, tanto desde el punto de vista del clima organizacional de los trabajadores así como la satisfacciónde los usuarios que acuden a los servicios de salud.

Agradecimiento: A la licenciada en estadística Alicia Bustamante López, por su asesoría 


\section{REFERENCIAS BIBLIOGRÁFICAS}

1. Flores-Ruiz E, Miranda-Novales MG, Villasís-Keever MÁ. El protocolo de investigación VI: cómo elegir la prueba estadistica adecuada. Estadistica inferencial. Rev Alerg Mex. 2017;64(3):364-370.

2. Mondragón M. Uso de la correlación de spearman en un estudio de intervención en fisioterapia. Mov. cient. 2014;8(1):98-104

3. Vela S. Clima organizacional y satisfacción de usuario externo - Hospital Regional Hermilio Valdi- zan - Huánuco - 2015 (Tesis Doctoral). Huánuco, Perú: Universidad de Huánuco; 2016.

4. Valdivia M. Efecto del clima organizacional en la satisfacción del usuario externo en el Centro Médico Universitario Pedro P. Diaz, Arequipa, 2017 (Tesis de Maestria). Arequipa, Perú: Universidad Nacional San Agustín de Arequipa; 2017.

5. Chincha E. Clima organizacional en relación a la satisfacción del usuario externo del "Hospital de Yungay"2016 (Tesis de Maestria). Huaraz, Perú: Universidad Nacional Santiago Antúnez de Mayolo; 2018.
6. Calderón M. Clima organizacional y satisfaccion de los usuarios del Centro de Salud Bellavista, Callao (Tesis de Maestría). Lima, Perú: Universidad Peruana de Ciencias Aplicadas; 2017.

7. Fernández-Oliva D, Revilla-Velásquez M, KolevicRoca L, Cabrejos-Castilla I, Muchaypiña-Gallegos I, Sayas-Avilés I, et al. Clima organizacional y satisfacción del usuario externo en los servicios de hospitalización del Instituto Nacional de Salud de Niño, 2017. An Fac med. 2019;80(2):188-92. DOI: https://doi.org/10.15381/anales.802.15745 